\title{
Predictions of the events of the satellites of Saturn during the 2009 equinox ${ }^{\star}$
}

\author{
J. E. Arlot and W. Thuillot
}

\begin{abstract}
Institut de mécanique céleste et de calcul des éphémérides - Observatoire de Paris, UMR 8028 du CNRS, 77 avenue Denfert-Rochereau, 75014 Paris, France, USTL, Lille, UPMC, Paris, France e-mail: [arlot; thuillot] @imcce.fr
\end{abstract}

Received 20 February 2008 / Accepted 19 March 2008

\section{ABSTRACT}

\begin{abstract}
Aims. The Saturnian equinox will occur in 2009; i.e., the Sun (and the Earth, very close to the Sun as seen from Saturn) will be in the equatorial plane of Saturn. Eclipses of the satellites by Saturn or mutual eclipses and occultations will occur among the seven first satellites orbiting in the Saturnian equatorial plane. This paper provides predictions of these events, along with information useful for observing them. Such events are uncommon, since they only occur every 15 years during the Saturnian equinox. The present dynamical models of motion of the Saturnian satellites include many effects on their motion. One needs accurate observations, such as mutual events, to determine all the relevant parameters.

Methods. For these predictions, we used the best theoretical models of the motion of the satellites, since the prediction of events is very sensitive to the accuracy of the relative calculated positions of the satellites.

Results. Therefore, we encourage professional and amateur astronomers to join the observers network to get as many observations as possible. Data on the predictions of the events and interactive software for their visibility are available on the web server of the IMCCE (www . imcce. fr/phemu09).
\end{abstract}

Key words. astrometry - occultations - eclipses - celestial mechanics - planets and satellites: individual

\section{Introduction}

The astrometric observations of the Satunian satellites provide data with an astrometric accuracy of 0.1 arcsec at best, which corresponds to $600 \mathrm{~km}$ because of the distance from Saturn to Earth. Only space probes can provide observations with better astrometric accuracy. However, we have had experience with regularly observed Galilean satellites, with the first observations of the events of the Saturnian satellites in 1995, and with the observations of the Uranian satellites events in 2007. They have shown that the photometric observations of mutual eclipses and occultations occurring during the equinox on Saturn may reach higher astrometric accuracy since results are obtained in kilometers with an accuracy of 6 to $60 \mathrm{~km}$ that corresponds to 0.001 to $0.01 \mathrm{arcsec}$. Recent results on the accelerations in the longitudes of the Galilean satellites have shown how such accurate astrometric observations may be a help for modeling the internal structure of the satellites (Lainey et al. 2005, 2007). This encouraged us to gather as many accurate observations as possible.

The mutual events of the Saturnian satellites were observed in 1980 and 1995 (Dourneau 1982; Aksnes et al. 1984; Thuillot et al. 2001), now we can take advantage of improvements in detectors that make the observations easier. Observations over long periods of time increases the possibility of obtaining results on the evolution of the orbits.

We plan to perform an observational campaign for the next occurrence of mutual events in 2009. For that, we use two different theoretical dynamical models of motion of the satellites

* Tables 4-7 are only available in electronic form at the CDS via anonymous ftp to cdsarc.u-strasbg.fr (130.79.128.5) or via http://cdsweb.u-strasbg.fr/cgi-bin/qcat?J/A+A/485/293 to predict these events in order to be certain which events will occur.

\section{The events}

Since the seven first satellites of Saturn have their orbits almost in the same plane, eclipses by the planet and mutual events will occur regularly when the Earth (for the occultations) and the Sun (for the eclipses) pass through the orbital plane of the satellites. These planes correspond closely to the equatorial plane of Saturn. This occurrence takes place at the equinox on Saturn, i.e. when the planetocentric declination of the Sun (and the Earth, which appears to be close to the Sun as seen from Saturn) are near zero. Figure 1 shows the variation of these declinations for the equinoxes of 1995 and 2009: the best periods for large, deep, mutual events appear to be May 2009 for the occultations and July 2009 for the eclipses. Note that on September 4, when the Earth will transit in the equatorial plane of Saturn, the rings will seem to disappear making easy the observation of the small satellites near the planet. The opposition of Saturn with the Sun occurs in March 2009 and the equinox will occur on August 12 near the conjunction Saturn-Sun. This is not very favorable: the observations being possible only before and after the equinox.

Descriptions of the mutual eclipses and occultations have been made in previous papers, especially in technical notes made for the former occurrences $(2003,1997$, etc.) available on the web site of IMCCE at the address http://www.imcce.fr/ phemu09 or in Arlot (1999). Four types of events will occur.

- Eclipses by the shadow of the planet Saturn. The atmosphere of Saturn will degrade the accuracy of the measurement of 

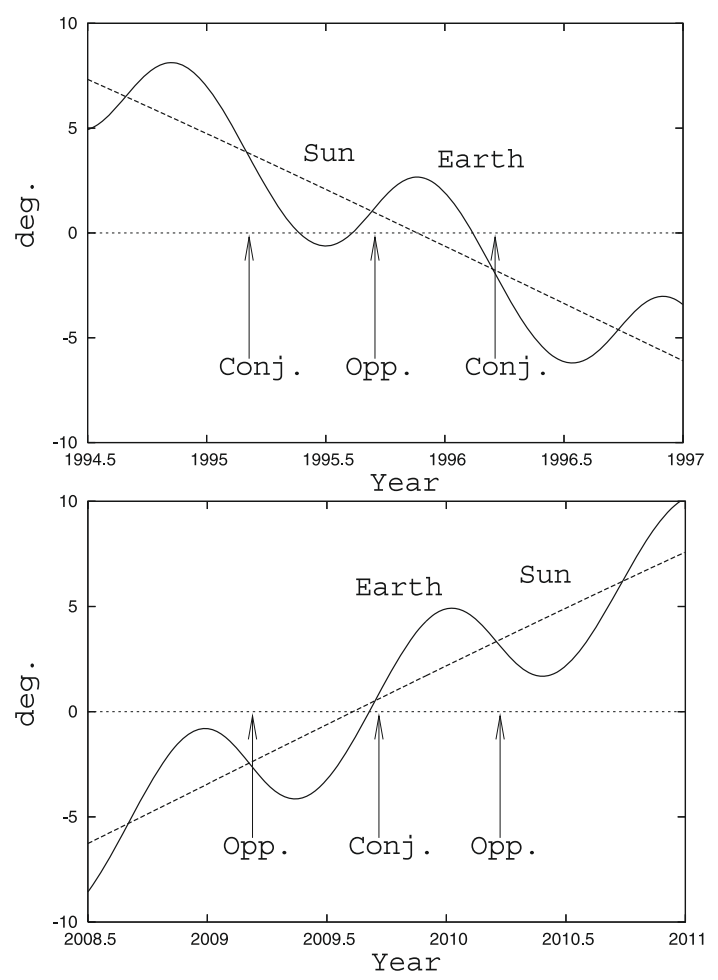

Fig. 1. Saturnicentric declination of the Earth and the Sun in 1995 and 2009.

the time of the event but will remain sufficient for astrometric purposes; but be careful, because some events may be polluted by the brightness of the rings. Similar events are the transits of the shadows of the satellites on the disk of the planet. These events are not scientifically interesting but the transits of the shadow of Titan are spectacular: the transits occur from February 24, 2009 to January 9, 2010. See the web server http: //Www. imcce. fr/phemu09 for dates and visibility.

- Occultations of the satellites by the planet and transits of the satellites on the disk of the planet. These events are difficult to analyze because it is impossible to model the figure of the planet with sufficient accuracy.

- Mutual occultations. These consist of the arrival of the disk of the occulting satellite on that of the occulted satellite; therefore, the amount of light received by a terrestrial observer decreases and increases during the event. The absence of astmosphere on the satellites (except Titan) will make these observations very accurate.

- Mutual eclipses, corresponding to the arrival of the eclipsed satellite in the shadow cone of the eclipsing satellite. In this case, only the eclipsed satellite may be observed, leading, in some cases, to a total eclipse (the complete disappearance of the eclipsed satellite).

During the mutual events, the magnitude of the events will be small at the beginning and the end of the period. The planetocentric declinations of the Earth and the Sun are not yet zero and the satellites are not exactly on the same line as seen from the Earth or from the Sun. On the other hand, the magnitude of the events will be greater for the zero value of the planetocentric declination of the Earth and the Sun. Observers should be aware that the signal-to-noise ratio will be higher for deep phenomena. In contrast, the eclipses by Saturn correspond to the total disappearance of a satellite in the shadow of the planet or to a reappearance. If the disappearance is easy to observe (just record the decreasing magnitude of the satellite), the reappearance is more difficult because we must know in advance where the satellite will appear, with its light flux starting from zero. A two-dimensional receptor is then necessary.

\section{The predictions for $\mathbf{2 0 0 8 - 2 0 1 0}$}

\subsection{The dynamical models used}

Prediction of the events requires an accurate model for the motion of the satellites, since the occurrence of the mutual events is very sensitive to the small inclinations of the orbits of the satellites to the equatorial plane of Saturn. A difference of a few tenths of an arcsecond ( 0.1 arcsec corresponds to $600 \mathrm{~km}$ near Saturn) in the relative positions of the satellites may determine whether the event will occur or not. Therefore, we use the accurate ephemerides TASS proposed by Vienne \& Duriez (1995). The TASS ephemerides now have a drift reaching about $100 \mathrm{~km}$, more recent ephemerides such as SAT252 from JPL (Jacobson 2006), fitted on recent data from Cassini, are more accurate. However, the differences in the predictions are small, with the magnitude drops slightly different. The times of maximum of magnitude drop drift of 10 to $30 \mathrm{~s}$ of time. The use of more accurate ephemerides will be useful for reducing the data. We also provide data calculated with the Do93 ephemerides (Dourneau 1993), which were used for the former predictions. All these ephemerides are available on the Web site of IMCCE at http: // wWw. imcce. fr/sat.

The calculations for predicting the events differ for the occultations and for the eclipses. Explanations have already been given in earlier papers providing predictions of such events (Arlot 2008; Thuillot \& Arlot 1996). Some events are predicted with low accuracy, when the relative velocity of the two satellites or of the eclipsed satellite with reference to the cones is very small, or if there are non linear variations. What are the accuracies of these predictions? Since we used two different dynamical models fitted on different sets of observations, the differences in the predictions are a good estimate of the accuracy of the predictions. In Tables 4 to 7, the difference between Cols. 6 and 7 provides the accuracy for the date of the maximum of the event. The difference between Cols. 13 and 14 provides the accuracy in magnitude drop, and the difference between Cols. 15 and 16 the accuracy on the duration of the event. The prediction using TASS ephemerides would be nearer the true value.

\subsection{The mutual events data}

All the possible events are available on the web site of the IMCCE at the address http://www. imcce.fr/phemu09. Tables 4 to 7 provide the detailed data of the main events (events more easily observable i.e. occurring at more than one planetary radius from the limb of the planet (outside the ring) and having a magnitude drop over 10 percent. These events are first calculated with the TASS ephemeris. Some data are also provided calculated with the Do93 ephemerides (as indicated below).

Column 1: date of the maximum of the event.

Column 2: nature of the event: 1 OCC 2 means that satellite 1 occults satellite 2, 3 ECL 4 means that satellite 3 eclipses satellite 4, etc.; P stands for partial, A for annular, T for total, and a blank means an eclipse by the penumbra.

Column 3: (only for eclipses): h, min, s in TT of the beginning of the eclipse by the penumbra. 
Column 4: h, min, s in TT of the beginning of the eclipse by the shadow or beginning of the occultation.

Column 5: (only for total events) h, min, s in TT of the beginning of totality.

Column 6: h, min, s in TT of the maximum of the event.

Column 7: h, min, s in TT of the maximum of the event calculated with Do93 ephemeris.

Column 8: (only for total events) h, min, s in TT of the end of totality.

Column 9: h, min, $\mathrm{s}$ in TT of the end of the eclipse by the shadow or end of the occultation.

Column 10: (only for eclipses): h, min, s in TT of the end of the eclipse by the penumbra.

Column 11: flux drop in the $V$-band (if 0 , no event, but a small magnitude drop may be recorded due to the uncertainties of the ephemerides; if 1 , total event).

Column 12: flux drop with the same albedoes for all the implied satellites (if 0 , no event, but a small magnitude drop may be recorded due to the uncertainties of the ephemerides; if 1 , total event).

Column 13: magnitude drop with the same albedoes for all the implied satellites (if 0 , no event, but a small magnitude drop may be recorded due to the uncertainties of the ephemerides; if very large, total event).

Column 14: magnitude drop with the same albedoes for all the implied satellites (if 0 , no event, but a small magnitude drop may be recorded due to the uncertainties of the ephemerides; if very large, total event) calculated with Do93 ephemeris.

Column 15: duration of the event in seconds; no duration is indicated for grazing events. The eclipse inside the penumbra is not taken into account in the duration.

Column 16: duration of the event in seconds calculated with Do93 ephemeris; no duration is indicated for grazing events. The eclipse inside the penumbra is not taken into account in the duration.

Column 17: apparent distance from the occulted or eclipsed satellite to the center of Saturn in saturnian radii. If this distance is less than 1, it means that the event occurs in front of the planet Saturn. This is a nice observation - especially the eclipses - with a good seeing, but without any scientific interest! These events are only available from the web site.

Column 18: impact parameter in arcsec (apparent distance between the centers of the satellites for the occultations and from the eclipsed satellite to the axis of the shadow cone for the eclipses).

Column 19: impact parameter in arcsec (apparent distance between the centers of the satellites for the occultations and from the eclipsed satellite to the axis of the shadow cone for the eclipses) calculated with Do93 ephemeris.

The dates are provided in terrestrial time (TT) since the UTC for this period is not yet available. The difference TT - UTC will, however, be near $65 \mathrm{~s}$ in 2009. Observations will be recorded with reference to UTC.

In the list of events in Tables 4 to 7 , some events will occur during an eclipse by Saturn: we removed these events from the list but, for the following events, an eclipse occurs before the mutual event so that the eclipsed satellite will not be visible a long time before the mutual event.

- On June 10, 2009, Mimas (S-1) eclipses Tethys (S-3) from $22^{\mathrm{h}} 37^{\mathrm{m}} 44^{\mathrm{s}}$ to $22^{\mathrm{h}} 38^{\mathrm{m}} 54^{\mathrm{s}}$ TT and S-3 (Tethys) is eclipsed until $20^{\mathrm{h}} 46^{\mathrm{m}} 23^{\mathrm{s}}$ TT.
Table 1. Albedoes used for the calculation of the magnitude drop.

\begin{tabular}{lllc}
\hline \hline & Satellites & NASA & Morrison \\
\hline S-1 & Mimas & 0.60 & 0.77 \\
S-2 & Encelade & 1.00 & 1.04 \\
S-3 & Tethys & 0.80 & 0.80 \\
S-4 & Dione & 0.70 & 0.55 \\
S-5 & Rhea & 0.70 & 0.65 \\
S-6 & Titan & 0.22 & 0.20 \\
S-7 & Hyperion & 0.30 & 0.25 \\
\hline
\end{tabular}

- On June 26, 2009, Mimas (S-1) eclipses Enceladus (S-2) from $1^{\mathrm{h}} 2^{\mathrm{h}} 35^{\mathrm{s}}$ to $1^{\mathrm{h}} 3^{\mathrm{m}} 31^{\mathrm{s}}$ TT and $\mathrm{S}-2$ (Enceladus) is eclipsed until June $25,23^{\mathrm{h}} 47^{\mathrm{m}} 19^{\mathrm{s}}$ TT.

The magnitude drops are provided in several forms: first the flux drop in unit of flux $(0=$ no decrease of light; 1 = total disappearance of the object) and second in magnitude units that are more understandable for human eyes $(0=$ no decrease in light, unchanged magnitude of the object(s); $n=$ increase in magnitude at the maximum of the event). We calculated the drop depending on the albedoes of the implied satellites (for the occultations) in the $V$-band, which is commonly used by observers. Table 1 provides the values for the albedoes. They are taken from Morrisson \& Morrison (1977) for a phase not equal to zero. They have very little influence on the results (and of course do not influence the magnitude drop of the eclipses at all) and are not very different in the other bands. We also provide the flux drop without albedoes i.e. with the same albedoes for the four satellites.

During the 2009-2010 event season, 441 events have been predicted (occurring from September 9, 2008 to June 18, 2010). Not all of them are observable: some will remain grazing with no detectable signal, some will occur in front of or behind Saturn, or during an eclipse by the planet. Some will also be difficult to observe because of the conjunction of Saturn with the Sun, but we did not eliminate these events from Tables 4 to 7 . Only 123 - easily observable as indicated in Sect. 3.2 - are presented here (occurring from October 2, 2008 to June 18, 2010). In the next sections, we provide more information on the visibility and on the observability of these events.

\subsection{The eclipse data}

Besides the mutual events, eclipses of the satellites by Saturn will occur. Though the astrometric and photometric accuracy of their observation is poorer than that of the mutual events, we provide the list of the more easily observable eclipses. These eclipses provide data with an accuracy similar to what is obtained with direct astrometric imaging. In several papers, Mallama (2000, 2007a,b) give models for that kind of event for the Jovian, Neptunian, and Uranian systems. Tables 2 and 3 provide the list of the easily observable eclipses by Saturn, i.e. occurring at more than 10 arcsec from the limb of Saturn (avoiding light pollution by the rings). We also eliminate the eclipses occurring when Saturn is less than 10 degrees from the Sun. These criteria explain the small number of observable eclipses. These events were calculated with TASS ephemerides. Tables 2 and 3 provide:

Column 1: date of the maximum of the event in TT.

Column 2: hour, minute, second of the maximum of the event in TT.

Column 3: the half-duration of the eclipse, i.e. the time needed for the half-disappearance or reappearance of the satellite in seconds of time (negative values for the reappearances). 
Table 2. Eclipses of Titan (S-6) by the planet Saturn.

\begin{tabular}{|c|c|c|c|c|c|c|}
\hline (1) & (2) & (3) & (4) & (5) & (6) & (7) \\
\hline \multicolumn{7}{|l|}{2009} \\
\hline 0405 & 133732 & 729 & 10 & 1.7 & -1.1 & $\mathrm{R}$ \\
\hline 0421 & 131012 & 636 & 15 & 2.3 & -1.2 & $\mathrm{R}$ \\
\hline 0507 & 123924 & 577 & 18 & 2.7 & -1.2 & $\mathrm{R}$ \\
\hline 0523 & 120554 & 538 & 20 & 2.9 & -1.2 & $\mathrm{R}$ \\
\hline 0608 & 11303 & 511 & 20 & 3.0 & -1.1 & $\mathrm{R}$ \\
\hline 0624 & 105159 & 492 & 18 & 3.0 & -0.9 & $\mathrm{R}$ \\
\hline 0710 & 101147 & 481 & 16 & 2.8 & -0.8 & $\mathrm{R}$ \\
\hline 0726 & 92926 & 476 & 13 & 2.5 & -0.5 & $\mathrm{R}$ \\
\hline 1029 & 231426 & 587 & 11 & -2.0 & 1.0 & D \\
\hline 1114 & 224021 & 650 & 14 & -2.3 & 1.2 & D \\
\hline 1130 & 220949 & 749 & 16 & -2.5 & 1.4 & D \\
\hline 1216 & 214451 & 932 & 18 & -2.5 & 1.6 & D \\
\hline 1217 & 10849 & 934 & 11 & -1.5 & 1.6 & $\mathrm{R}$ \\
\hline \multicolumn{7}{|l|}{2010} \\
\hline 0101 & 213123 & 1432 & 17 & -2.4 & 1.7 & D \\
\hline 0101 & 234558 & 1440 & 13 & -1.7 & 1.7 & $\mathrm{R}$ \\
\hline
\end{tabular}

(1) Date of the eclipse: month, day;

(2) Date of the eclipse: h, min, s;

(3) Half-duration in seconds of time;

(4) Apparent distance to the limb of the planet in arcsec;

(5) X satellite/center of Saturn in Saturnian radii;

(6) Y satellite/center of Saturn in Saturnian radii;

(7) $\mathrm{D}=$ disappearance; $\mathrm{R}=$ reappearance.

Table 3. Eclipses of Hyperion (S-7) by the planet Saturn.

\begin{tabular}{|c|c|c|c|c|c|c|}
\hline (1) & (2) & (3) & (4) & (5) & (6) & (7) \\
\hline \multicolumn{7}{|l|}{2009} \\
\hline 0413 & 180748 & 37 & 14 & 2.2 & -1.1 & $\mathrm{R}$ \\
\hline 0505 & 35217 & 32 & 20 & 2.8 & -1.2 & $\mathrm{R}$ \\
\hline 0526 & 130942 & 30 & 22 & 3.2 & -1.2 & $\mathrm{R}$ \\
\hline 0616 & 215541 & 28 & 21 & 3.2 & -1.0 & $\mathrm{R}$ \\
\hline 0708 & 60634 & 28 & 18 & 3.0 & -0.8 & $\mathrm{R}$ \\
\hline 0729 & 133947 & 28 & 13 & 2.5 & -0.5 & $\mathrm{R}$ \\
\hline 1112 & 140628 & 44 & 14 & -2.3 & 1.4 & D \\
\hline 1203 & 192236 & 64 & 17 & -2.5 & 1.7 & D \\
\hline 1203 & 221300 & 64 & 12 & -1.6 & 1.7 & $\mathrm{R}$ \\
\hline
\end{tabular}

(1) Date of the eclipse: month, day;

(2) Date of the eclipse: h, min, s;

(3) Half-duration in seconds of time;

(4) Apparent distance to the limb of the planet in arcsec;

(5) X satellite/center of Saturn in Saturnian radii;

(6) Y satellite/center of Saturn in Saturnian radii;

(7) $\mathrm{D}=$ disappearance; $\mathrm{R}=$ reappearance.

Column 4: the apparent distances to the limb of Saturn in $\operatorname{arcsec}$.

Columns 5 and 6: the position of the satellite with respect to the center of Saturn in Saturnian radii. The knowledge of this position is essential finding the satellites when reappearing.

Column 7: phase of the eclipse: $\mathrm{D}$ for disappearance; $\mathrm{R}$ for reappearance.

\subsection{The visibility of the events}

Since they occur anytime and since their duration is usually only a few minutes, the events are observable only from certain sites where Saturn is visible, above the horizon at the time of the event. The declination of Saturn during these mutual events will be between 0 and 8 degrees. Therefore, both hemisphere are just as good for observations. Table 8 provides the number of events
Table 8. Visibility of the mutual events depending on the site of observation.

\begin{tabular}{lcc}
\hline \hline Observatories & $(1)$ & $(2)$ \\
\hline Itajuba (Brazil) & 17 & 53 \\
Yunnan (China) & 16 & 40 \\
Kavalur (India) & 15 & 48 \\
Rio de Janeiro (Brazil) & 15 & 52 \\
Mac Donald (USA) & 14 & 54 \\
Nanjing (China) & 14 & 41 \\
Irkutsk (Russia) & 14 & 26 \\
Canarian Islands (Spain) & 13 & 44 \\
Topeka, Kansas (USA) & 13 & 47 \\
Mauna Kea, Hawaii (USA) & 12 & 57 \\
Beijing (China) & 12 & 33 \\
La Silla - ESO (Chile) & 11 & 50 \\
Sydney (Australia) & 11 & 54 \\
Pic du Midi (France) & 11 & 41 \\
Barcelona (Spain) & 11 & 45 \\
Athens (Greece) & 11 & 42 \\
Pulkovo (Russia) & 10 & 31 \\
Torino (Italy) & 10 & 43 \\
Capetown (South Africa) & 10 & 49 \\
Bordeaux (France) & 10 & 40 \\
Haute Provence (France) & 10 & 41 \\
Paris (France) & 10 & 36 \\
Bucarest (Romania) & 9 & 36 \\
Dodaira (Japan) & 9 & 40 \\
Berlin (Germany) & 9 & 34 \\
Naucsny (Ukraine) & 8 & 32 \\
\hline
\end{tabular}

(1) Events easily observable: magnitude over 10 percent occurring at more than one saturnian radius from the limb of the planet; (2) All events.

observable from some selected sites around the world (calculated from the 131 events selected as easily observable (far from the disk and the rings, with a magnitude drop greater than 10 percent). All the possible events are provided on our web server through http://www . imcce. fr/phemu09. We considered that an event is observable when the Sun is more than 10 degrees below the horizon and Saturn is more than 15 degrees above the horizon. Table 9 provides the number of events. Grazing events may not be observable because of the sensitivity of these events to the inclinations of the orbital planes of the satellites. Their observability depends on the possible error in the ephemerides. As a result, if a signal is detected during an event, one will get valuable information about the ephemerides. Note also that the total or annular events will present light curves with a flat step allowing the analysis of the scattering law of the light on the surfaces of the satellites.

Short events or faint events (the flux drop of which being less than ten percent) will be difficult to catch because of the low signal-to-noise ratio, and observers will need near perfect photometric conditions. The long events (one hour or more) are interesting, too, but observers must take the changing elevation of Saturn on the sky into account: the event must end before Saturn sets. The observers should be aware that the predicted times may have an error of a few minutes in some cases, even if they are within a few seconds most of the time. This difference between the observation and the prediction contains information relevant to improving the theoretical models of the motions of the satellites. Therefore, the observations should start at least $10 \mathrm{~min}$ before the predicted beginning and must be referred to UTC within less than one tenth of a second. 
Table 9. Distribution of the events.

\begin{tabular}{|c|c|c|c|c|c|c|c|}
\hline \multicolumn{2}{|c|}{ Dates } & \multicolumn{2}{|c|}{ Number } & \multicolumn{2}{|c|}{ of } & \multicolumn{2}{|c|}{ events } \\
\hline & & (1) & (2) & (3) & (4) & $(5)$ & $(6)$ \\
\hline \multirow[t]{4}{*}{2008} & Sept. & 1 & 0 & 0 & 0 & 0 & 10 \\
\hline & Oct. & 4 & 1 & 0 & 1 & 0 & 36 \\
\hline & Nov. & 3 & 0 & 0 & 0 & 0 & 63 \\
\hline & Dec. & 23 & 9 & 0 & 9 & 0 & 92 \\
\hline \multirow[t]{12}{*}{2009} & Jan. & 19 & 9 & 1 & 8 & 0 & 123 \\
\hline & Feb. & 6 & 2 & 1 & 1 & 0 & 155 \\
\hline & Mar. & 7 & 1 & 1 & 0 & 0 & 172 \\
\hline & Apr. & 8 & 3 & 3 & 0 & 3 & 140 \\
\hline & May & 25 & 4 & 4 & 0 & 4 & 110 \\
\hline & June & 56 & 13 & 13 & 0 & 3 & 81 \\
\hline & July & 127 & 40 & 39 & 1 & 4 & 54 \\
\hline & Aug. & 85 & 31 & 21 & 10 & 0 & 28 \\
\hline & Sept. & 38 & 7 & 5 & 2 & 0 & 3 \\
\hline & Oct. & 16 & 4 & 4 & 0 & 1 & 24 \\
\hline & Nov. & 5 & 0 & 0 & 0 & 3 & 51 \\
\hline & Dec. & 1 & 1 & 1 & 0 & 4 & 80 \\
\hline \multirow[t]{6}{*}{2010} & Jan. & 1 & 0 & 0 & 0 & 2 & 110 \\
\hline & Feb. & 2 & 1 & 1 & 0 & 0 & 141 \\
\hline & Mar. & 0 & 0 & 0 & 0 & 0 & 173 \\
\hline & Apr. & 4 & 1 & 0 & 1 & 0 & 154 \\
\hline & May & 6 & 2 & 0 & 2 & 0 & 123 \\
\hline & June & 4 & 2 & 0 & 2 & 0 & 94 \\
\hline
\end{tabular}

(1) Total number of events even not observable;

(2) Number of events easily observable;

(3) Number of mutual eclipses easily observable;

(4) Number of mutual occultations easily observable;

(5) Number of eclipses easily observable;

(6) Mean apparent distance Sun-Saturn in degrees.

Information on the visibility of the events from any site of observation is provided on the web site of IMCCE at the address http: //www .imcce.fr/phemu09.

Note that the equinox on Saturn occurs on August 12 near the conjunction Saturn-Sun, which occurs on September 18. This makes many of the events unobservable.

\section{The observations of the events}

\subsection{The receptors}

The observation of the mutual events consists in recording the light flux as a function of time using a photometric receptor. The observation of the eclipses by Saturn is the same. At the present time, CCDs are the most common detectors for this type of observation.

- The speed of the acquisition of the images allows the recording of at least 3 images per second (for short events);

- Each image must be dated in UTC within an accuracy better than $0.1 \mathrm{~s}$ in order to obtain an astrometric accuracy of $1 \mathrm{~km}$ for the relative position of the satellites;

- For the eclipses, we do not know exactly where the satellite will reappear, so the two-dimensional CCD will not miss the reappearance of the satellite;

- Another advantage of two-dimensional receptors is the possibility of obtaining several satellites in the field. This will help for the photometric calibration.

Video cameras running at 25 frames per second may also be used, but the faintness of the satellites makes it necessary to use a large telescope. However, do record the UTC time on each image.
Other types of detectors may be used, such as single channel photoelectric photometers for fast photometry in several spectral bands. Any telescope, no matter small, may be used: the stability of the image and a good guidance of the instrument is necessary, however. With small telescopes, it will not be possible to get more than one image per second.

Technical notes on the use of several types of detectors are available by request from the author or on the web server of IMCCE at the address http: //www. imcce. fr/phemu09.

\subsection{The calibration}

Most of the time, the raw light curves are difficult to analyze without a specific photometric reduction. Since we only need relative photometry (the recorded magnitude drop is measured by referring to the magnitude of the satellites before and after the event). However, it is important to record a reference object not affected by the event at the same time. Using a twodimensional receptor will allow the observer to simultaneously record the satellites involved in the event, and another satellite not involved, with constant brightness (at least during the event). Only for long events will it be useful to take the rotation of the satellites into account. A two-dimensional detector will also record the sky background simultaneously. The reduction will then easily solve the problems related to light clouds or to the variations in the elevation of the observed bodies in the sky, as well as to the brightness of the sky background before sunrise or after sunset. If using a single channel receptor, the observer will need to alternatively record the sky background and a reference object either during the event, for a long event (more than $20 \mathrm{~min}$ ), or just before and after the event for a short event.

\subsection{The PHEMUO9 campaign of observations}

In order to optimize the observations and to collect the maximum number of phenomena, we are organizing a campaign of observations to coordinate of the observing sites as we have done previously, leading to publication of a very complete catalog of data such as in Thuillot et al. (2001) for the 1995 occurrence, subsequently analyzed by Noyelles et al. (2003). This database is available at the address: http: //WWW.imcce. fr/fr/ephemerides/donnees/ $\mathrm{nsdc} / \mathrm{nsdf} / \mathrm{fsaphemu}$.html. The reader is encouraged to join our campaign of observations, to contact the author at arlot@imcce.fr, and to get information on the campaign of observations from the web address http://www.imcce.fr/ phemu09.

\section{The interest of these observations}

\subsection{Astrometry of the main satellites of Saturn}

The main goal of the observation of the mutual phenomena of the main satellites of Saturn is to obtain astrometric data leading to relative positions of the satellites with high accuracy. Observations of the mutual events have been shown to be more accurate than photographic or CCD direct observations of astrometric positions. The measurement of the magnitude drop at the time of the minimum distance allows this minimum distance to be determined. Thanks to the absence of an atmosphere on the satellites, the observations of mutual events are more interesting than those of the eclipses by Saturn because of the sharpness of the light curves. Observations of eclipses by Saturn led to relative positions determined within an accuracy similar to 
photographic or CCD positions (around $600 \mathrm{~km}$ ) and mutual events to an accuracy better than $100 \mathrm{~km}$. This accuracy for the mutual events may be improved to $20 \mathrm{~km}$ or more after using a more sophisticated reduction involving surface reflectivity.

Such observed positions help for fitting the dynamical models of the motions of the satellites. Their accuracy allows better determination of the eccentricities that should constrain the model of tidal effects on Enceladus and the model of the interior of the satellites. Also, these observations allow detection of an acceleration in longitude, which is a signature of the tides. Space probes have made some precise observations over an interval of time that is too short to be able to determine an acceleration. For that, we need more accurate observations over a long interval of time, as provided by the mutual events and the space probes together. At the present time, the space probe Cassini is providing data on the Saturnian system. If it continues during the equinox period, the Cassini data will be very useful for determining parameters through the inversion of the light curves of the mutual events, allowing better reduction of the old mutual events observed in 1995 and 1980.

\subsection{Planetology: analysis of the surfaces}

Looking for a better reduction of the mutual phenomena, Vasundhara et al. (2003) and Emelianov et al. (2000) for the Galilean satellites, have demonstrated that a new reduction considering that the satellites are not uniform disks may help to improve the accuracy of the data. Surface effects are non-negligible when observing the mutual events presenting light curves with an asymmetrical aspect. Taking the surface effects into account for the reduction allows determination of unknowns related to planetologic parameters such as the porosity and the rugosity of the surfaces of the satellites themselves measured here for low phase angles, involving different scattering laws modifying the observed light curves.

\section{Conclusion}

The occurrence of the mutual events in 2008-2010 is favorable because the declination of Saturn is between 0 and 8 degrees, allowing one to observe either in the northern hemisphere or in the southern one. Unfortunately, the opposition Saturn-Sun occurs too early in the year 2009 for a good observability of the events. Therefore, we need to concentrate efforts on all the events visible from each observation site.

We encourage observers to make as many observations as possible during this occurrence in order to complete the collection of data gathered since 1980. Thirty years of such accurate data will allow a major improvement in our knowledge of the dynamics of the Saturnian satellites.

Acknowledgements. This work was made possible thanks to the CNRS (Centre National de la Recherche Scientifique) and the Institut de mécanique céleste/ Observatoire de Paris.

\section{References}

Aksnes, A., Franklin, F., Millis, R., et al. 1984, AJ 89, 280

Arlot, J. E. 1999, Proceedings of the 5th workshop PHEMU97, held in Catania, Italy on March 4-6, 1997, ed. J.-E. Arlot, \& C. Blanco (Paris: IMC Editions), pp. 59-66, pp. $75-82$

Arlot, J. E. 2008, Prediction of the mutual events of the Galilean satellites of Jupiter in 2009, A\&A, 478, 285

Dourneau, G. 1982, A\&A, 112, 73

Dourneau, G. 1993, A\&A, 267, 292

Emelianov, N. V., Berejnoi, A. A., Vashkovjak, S. N., et al. 2000, A\&AS, 141, 433

Jacobson, R. A. 2006, SAT252 - JPL satellite ephemeris, JPL internal publication

Lainey, V., \& Tobie, G. 2005, New constraints on Io's and Jupiter's tidal dissipation, Icarus 179,485

Lainey, V., Arlot, J. E., Karatekin, O., \& Van Hoolst, T. 2007, in prep.

Mallama, A. 2000, Icarus, 147, 348

Mallama, A. 2007a, Icarus, 187, 620

Mallama, A. 2007b, Icarus, 192, 576

Morrison, D., \& Morrison, N. D. 1977, Photometry of the natural planetary satellites, in Planetary satellites (Tucson: University of Arizona Press), 363

Noyelles, B., Vienne, A., \& Descamps, P. 2003, Astrometric reduction of lightcurves observed during the PHESAT95 campaign of Saturnian satellites, A\&A, 401, 1159

Thuillot, W., \& Arlot, J.-E. 1996, Ann. Phys. 21, 47

Thuillot, W., Arlot, J.-E., Ruatti, C., et al. 2001, A\&A, 371, 343

Vasundhara, R., Arlot, J.-E., Lainey, V., \& Thuillot, W. 2003, A\&A, 410, 337

Vienne, A., \& Duriez, L. 1995, A\&A, 297, 588 